\title{
Micro-supercapacité à base de carbone nano-poreux
}

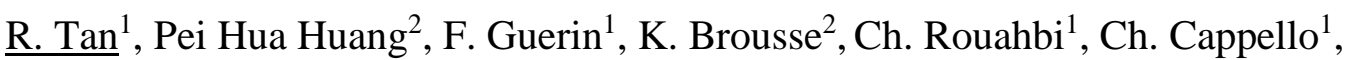 \\ P.-L. Taberna ${ }^{2}$, P. Simon ${ }^{2}$, F. Gessinn ${ }^{1}$, M. Respaud ${ }^{1}$ \\ ${ }^{1}$ AIME pôle CNFM de Toulouse, INSA de Toulouse (France) \\ ${ }^{2}$ CIRIMAT Toulouse (France) \\ ${ }^{3}$ INSA Atelier Mécanique, INSA Toulouse (France) \\ Contact e-mail : $\underline{\tan @ \text { aime-toulouse.fr }}$
}

\begin{abstract}
Cet article présente la mise en place d'une nouvelle formation dispensée à l'AIME de Toulouse, sur la thématique du stockage de l'énergie électrique sur silicium ( $\mathrm{Si}$ ), illustrant la fabrication et la caractérisation de supercapacités (SC) micrométriques. Ce stage a été dispensé pour des étudiants chimistes de Master 2 Erasmus MESC (Materials for Energy Storage and Conversion) de l'université de Paul Sabatier.
\end{abstract}

\section{1- Introduction}

Nous présentons la mise en place d'une nouvelle formation dispensée à l'AIME de Toulouse, sur la thématique du stockage de l'énergie électrique sur $\mathrm{Si}$, illustrant la fabrication et la caractérisation de supercapacités (SC) micrométriques. Ce stage a été dispensé pour des étudiants chimistes de Master 2 Erasmus MESC (Materials for Energy Storage and Conversion) de l'université de Paul Sabatier. Il peut cependant être facilement modulé afin d'intéresser des étudiants de niveaux inférieurs (IUT, Licence) ou de formations électroniciens/physiciens, du fait de sa facilité de mise en œuvre, tout comme convenir à des doctorants ou à des formations continues, compte tenu de la thématique abordée qui allie problématiques énergétiques et intégration de matériaux nanométriques en microélectronique, deux domaines de recherches en plein essor.

C'est dans ce contexte, et en particulier dans celui du stockage d'énergie dans l'électronique embarquée, que nous proposons aux étudiants d'élaborer et de caractériser des microsupercapacités à base de carbone activé. Le fonctionnement d'une SC repose sur l'accumulation de charge d'un électrolyte à la surface de deux électrodes actives. L'utilisation de carbone activé à haute surface spécifique en tant qu'électrodes conduit alors à des SC présentant de fortes densités de puissance $(>10 \mathrm{~kW} . \mathrm{kg}-1)$, avec une durée de vie quasi-illimitée étant donné l'absence de modification chimique des électrodes. Ce stage permet donc aux étudiants de se former dans différents domaines technologiques et scientifiques via : i/ la fabrication de puces supportant des collecteurs de courant (procédés conventionnels de micro-électronique), ii/ l'élaboration des électrodes de carbone activé (dépôt de couche mince par électrophorèse), et iii/ la caractérisation électrochimique des micro-supercapacités (par cyclo-voltamétrie).

\section{1- Fabrication des collecteurs de courant (1 journée)}

La première étape du stage concerne la réalisation des collecteurs de courant des micro-SC. Ils sont obtenus par photolithographie optique à partir de plaquettes de silicium 2 pouces. Les collecteurs sont en forme de peignes inter-digités (Figure 1-A/) permettant ainsi d'optimiser la surface des électrodes actives des SC. La séparation entre les deux collecteurs est de $160 \mu \mathrm{m}$ ou de $220 \mu \mathrm{m}$ selon les puces. 


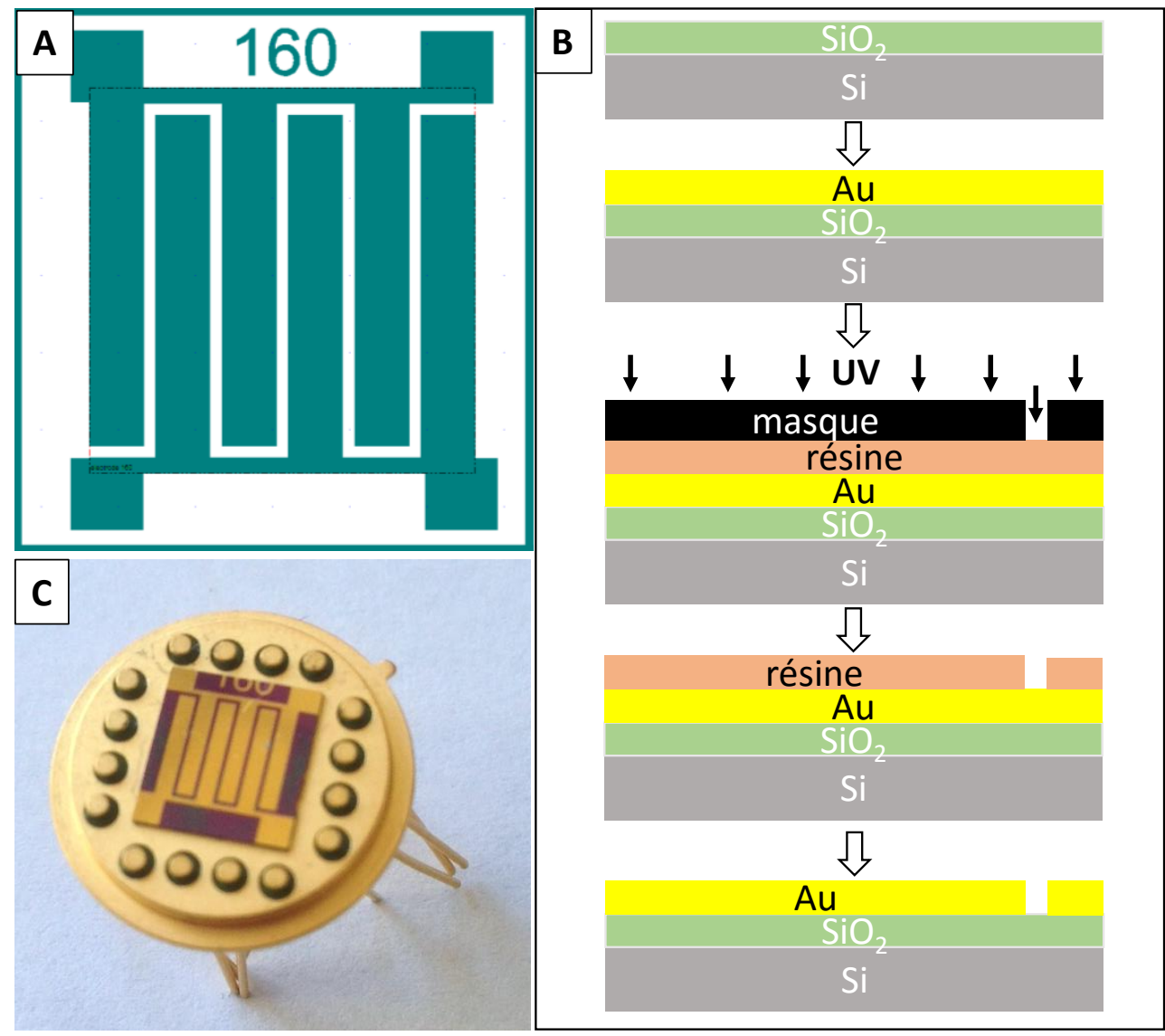

Fig.1. Elaboration du dispositif A/ design des électrodes en forme de peignes inter-digités. L'espacement entre deux électrodes est ici de $160 \mu \mathrm{m}$. En bleu : l'Au, en blanc le $\mathrm{SiO}_{2}$. B/ étapes de fabrication des électrodes : dépôt successif de couches minces de $\mathrm{SiO} 2$ et d' $\mathrm{Au}$, photolithographie du masque de $\mathrm{A} /$, gravure de l'or et dissolution de la résine. $\mathrm{C} /$ cliché optique d'une puce montée sur un boitier TO8.

Bien que la fabrication de ces collecteurs s'avère relativement aisée car elle ne nécessite qu'un seul niveau de masquage (Figure 1-B/), elle permet néanmoins aux étudiants d'avoir une vue complète sur des étapes clés de micro-électronique, en particulier la photogravure.

Les étudiants commencent donc par oxyder leurs plaquettes de silicium (400 nm). L'étape suivante consiste à déposer successivement des couches de Ti $(50 \mathrm{~nm})$ et d'Au $(250 \mathrm{~nm})$ par évaporation thermique. Une photolithographie des masques est ensuite réalisée sur les plaquettes préalablement enrésinées (résine positive). Les électrodes sont finalement obtenues après une attaque humide de 1 'or au $\mathrm{KI}_{2}$ et après dissolution de la résine (acétone). Un postrecuit thermique à $250{ }^{\circ} \mathrm{C}$ pendant 20 minutes est ensuite réalisé sur les plaquettes.

Les puces sont enfin découpées à la scie diamantée, puis montées et connectées sur un boitier type TO8 (Figure 1-C/).

\section{2- Elaboration des électrodes en carbone ( 1 jour):}

Le but de cette étape est d'élaborer les électrodes actives de la micro-SC. Le matériau choisi, du carbone activé YP-50F de la société Kuraray, a pour particularité de présenter une forte densité de nanopores (de taille inférieure à $2 \mathrm{~nm}$ ) qui conduit à une surface spécifique très élevée. Cela permet en outre à la SC de pouvoir adsorber un grand nombre de charges donnant lieu à de forte densité de puissance.

Nous avons choisi de déposer le carbone sur les collecteurs de courant par électrophorèse. Cette méthode présente dans notre cas plusieurs avantages : conservation de la morphologie du 
matériau, facilité de mise en œuvre (seule une source DC est requise), mais surtout, elle permet de bien localiser le dépôt sur la zone polarisée (les collecteurs dans notre cas).

Nous avons alors développé un montage dédié au dépôt par électrophorèse (Figure 2-A/). Il est constitué d'un système de guidage de boitiers TO8 connecté à une source de tension DC et de deux réservoirs métalliques pouvant être polarisés via des connecteurs électriques.

Le premier réservoir est rempli par une solution de carbone activé dilué dans un mélange d'éthanol, d'eau désionisée (DI), et de $\mathrm{MgCl}_{2}$. Ce sel a une double fonction: les ions $\mathrm{Mg} 2+$ adsorbés en surface du carbone facilitent l'attraction vers une anode, mais ils permettent également une meilleure accroche du carbone à l'interface du collecteur en or. Le second réservoir est rempli d'acétone et permet de rincer les puces, après le dépôt de carbone.

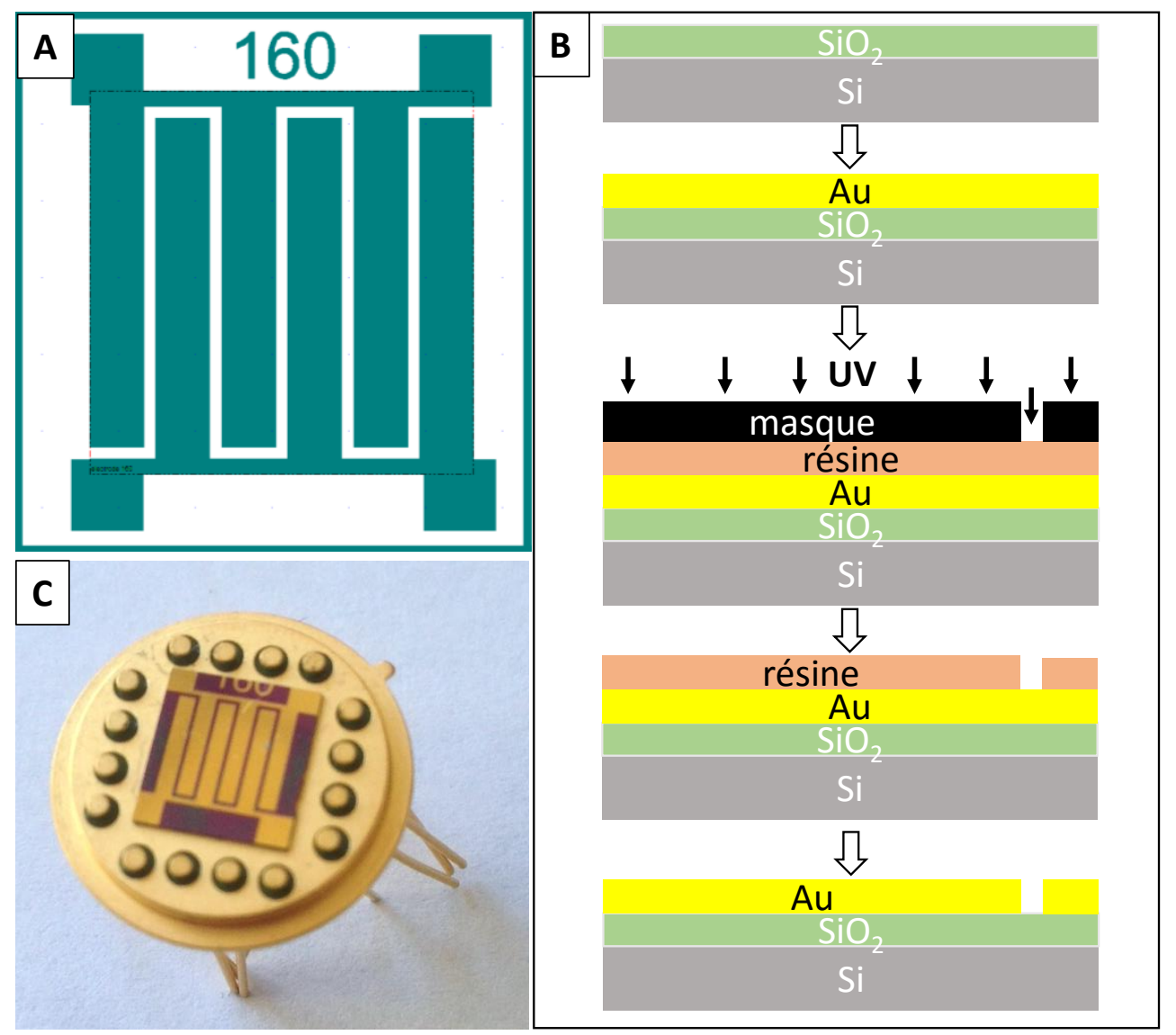

Fig.2. Elaboration du dispositif A/ design des électrodes en forme de peignes inter-digités. L'espacement entre deux électrodes est ici de $160 \mu \mathrm{m}$. En bleu : l'Au, en blanc le $\mathrm{SiO}_{2}$. B/ étapes de fabrication des électrodes : dépôt successif de couches minces de $\mathrm{SiO} 2$ et d' $\mathrm{Au}$, photolithographie du masque de $\mathrm{A} /$, gravure de l'or et dissolution de la résine. C/ cliché optique d'une puce montée sur un boitier TO8.

Le protocole de dépôt est le suivant. Dans un premier temps, les puces connectées sur TO8 sont nettoyées dans un bain piranha $\left(\mathrm{H}_{2} \mathrm{O}_{2}+\mathrm{H}_{2} \mathrm{SO}_{4}\right)$ pendant 2 minutes, puis rincées à l'eau DI et séchées à l'azote. Elles sont ensuite placées dans une étuve à $120^{\circ} \mathrm{C}$ pour évaporer les traces d'eau résiduelles. Les boitiers sont ensuite montés sur le banc expérimental de dépôt, et connectés à un générateur de tension DC (Figure 2-B/). Les deux collecteurs de courant sont portés au potentiel négatif et les réservoirs métalliques des bains de carbone et d'acétone au potentiel positif. Les boitiers sont ensuite immergés, «tête » vers le bas, dans le premier bain pour le dépôt électrophorétique. Une tension de $50 \mathrm{~V}$ continue est appliquée durant 10 secondes. Les boitiers sont ensuite plongés de la même manière dans le second bain (acétone) tout en maintenant la tension appliquée. Cette étape de rinçage est cruciale car elle permet de nettoyer 
le carbone qui peut se déposer en dehors des collecteurs de courant, notamment entre les peignes inter-digités, ce qui donnerait lieu à un court-circuit de la SC. Une micro-SC après dépôt et rinçage est présentée sur la Figure 2-C/.

Les boitiers sont finalement placés dans une étuve à $250{ }^{\circ} \mathrm{C}$ pendant 4 heures afin de consolider l'accroche du carbone sur les collecteurs.

\section{3- Caractérisation électrochimique des micro-supercapacités (1/2 journée)}

Les micro-SC obtenues sont ensuite caractérisées à l'aide d'un potentiostat/galvanostat VSP 300 de Bio-logic (Figure 3-A/). Les mesures sont effectuées en configuration deux électrodes (mesure du courant à travers la micro-SC en fonction d'un potentiel électrique (E), sans électrode de référence), après le dépôt d'une goutte d'un électrolyte organique sur la puce.

L'électrolyte choisi dans notre cas est composé d'un sel de tetraéthylammonium tétrafluoroborate (NET4BF4) dilué dans un solvant organique, le carbonate de propylène (PC).

Une mesure de voltamétrie cyclique du courant en fonction du potentiel $\mathrm{I}=\mathrm{f}(\mathrm{E}) \mathrm{d}$ 'une microSC est présentée dans la Figure 3-B/. Elle a été obtenue pour un balayage de $100 \mathrm{mV} / \mathrm{s}$ et une tension maximale appliquée de $1 \mathrm{~V}$.
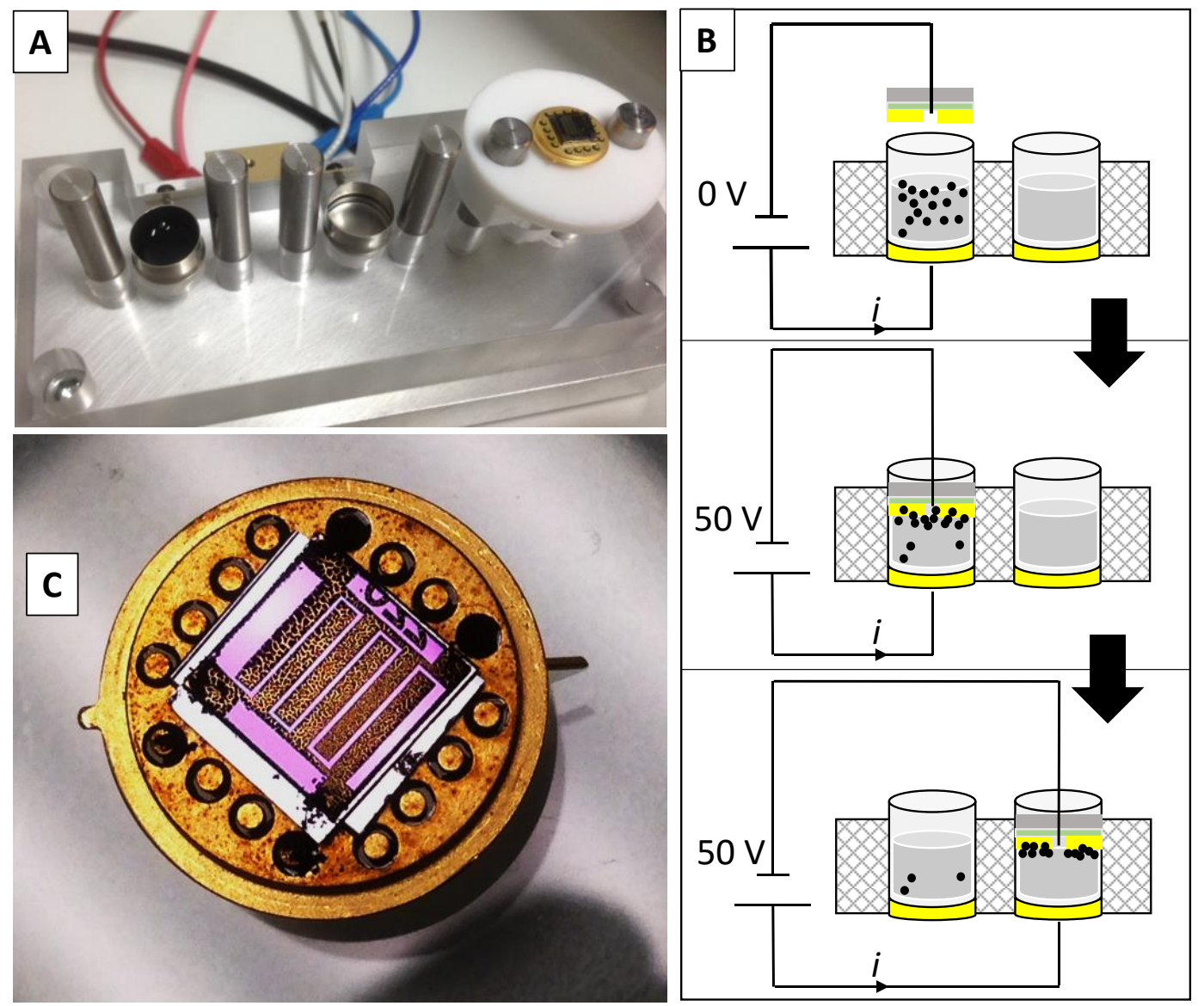

Fig.3. Intégration du carbone nanoporeux : A/ banc expérimental d'intégration du dépôt de carbone activé. Deux réservoirs métalliques accueillent respectivement la solution de carbone activé et de l'acétone. Les réservoirs sont reliés à des connecteurs électriques afin de les polariser. B/ schéma de principe du dépôt de carbone obtenu par électrophorèse. Les collecteurs sont portés au potentiel négatif, et les réservoirs au potentiel positif. Les électrodes sont immergées dans la solution de carbone dans un premier temps. Puis une tension continue de $50 \mathrm{~V}$ DC est appliquée pendant 10 secondes. Les échantillons sont ensuite plongés dans un bain d'acétone (étape de rinçage), toujours en présence du champ électrique (10 secondes) $\mathrm{C}$ / Cliché optique des dispositifs après dépôt et après rinçage. 
Le comportement capacitif, clairement visible sur les cycles, se traduit par une allure rectangulaire. Les phénomènes de charge et de décharge proviennent de l'adsorption et de la désorption des ions NET4+ et BF4- accumulés dans les nanopores des électrodes portées aux potentiels opposés. Ces cycles de charges/décharges peuvent également être visualisés sur le tracé du courant en fonction du temps (Figure 3-C/). Les capacités des dispositifs sont ensuite déduites par intégration du courant à l'aide du logiciel fourni avec le VSP 300. Les micro-SC présentent typiquement des capacités comprises entre 1-1.2 $\mathrm{mF}(\sim 5 \mathrm{mF} / \mathrm{cm} 2)$, en fonction du taux de recouvrement et de l'épaisseur du carbone déposé sur les collecteurs.

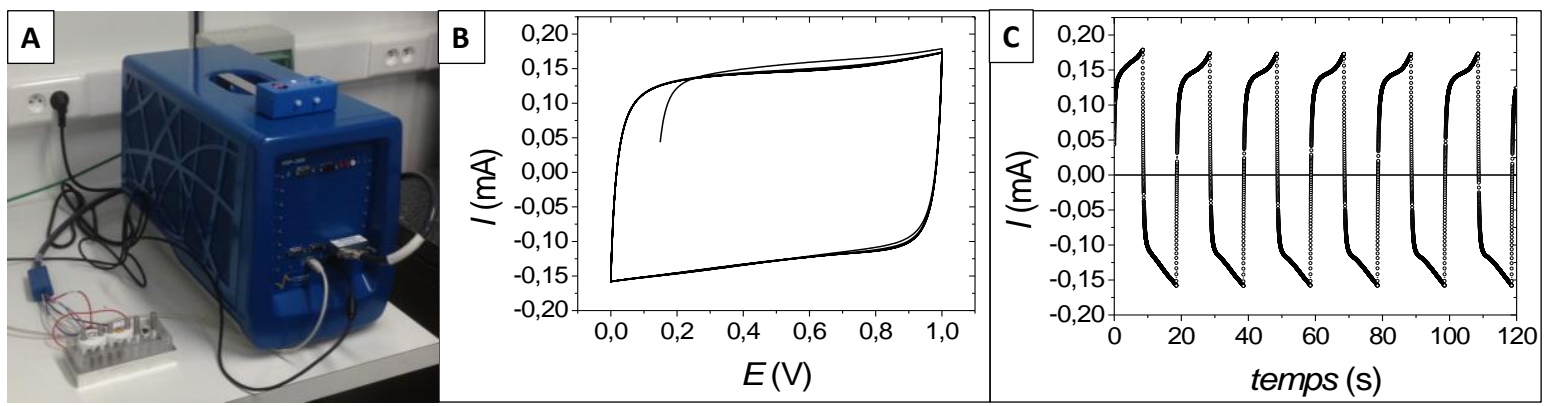

Fig.4. Caractérisation des capteurs A/ banc de mesure électrochimique des micro-supercapacités. Caractéristique de voltamétrie cyclique : $B / I=f(E)$ d'une micro-supercapacité et $C / I=f(t)$.

\section{4- Conclusion}

En conclusion, nous avons développé un nouveau stage permettant aux étudiants de fabriquer des supercapacités micrométriques à base de carbone activé. Cette formation possède un fort potentiel attractif envers de nombreuses filières, en particulier pour celles spécialisées dans le domaine des matériaux, du fait de sa facilité de mise en œuvre. Elle permet également aux étudiants de fabriquer et de caractériser dans son intégralité, un dispositif visant une application concrète dans l'électronique embarquée, et intégrant des matériaux à base de carbone nano-structuré.

\section{Remerciements}

La création de ce TP innovant a reçu le soutien du GIP-CNFM et du projet IDEFI-FINMINA ANR 2011-IDFI-0017 dans le cadre du programme Grands Investissements d'Avenir (PIA1). 